\title{
Silencing the receptor of activated C-kinase 1 (RACK1) suppresses tumorigenicity in epithelial ovarian cancer in vitro and in vivo
}

\author{
YANG LIN, MANHUA CUI, HONG TENG, FENGWEN WANG, WEI YU and TIANMIN XU \\ Department of Gynaecology and Obstetrics, The Second Hospital of Jilin University, Changchun, Jilin 130041, P.R. China
}

Received November 11, 2013; Accepted December 18, 2013

DOI: $10.3892 / \mathrm{ijo} .2014 .2274$

\begin{abstract}
Epithelial ovarian cancer (EOC) is a fatal disease for women due to lack of effective diagnostic biomarkers and therapeutic targets. Thus, it is important to identify and develop specific markers to formulate novel therapeutic methods for advanced and recurrent ovarian cancer. We found that the receptor of activated C-kinase 1 (RACK1) was elevated in most EOCs compared to normal ovarian tissue, and its expression levels correlated with key pathological characteristics including clinical stage and metastasis by quantitative PCR and immunohistochemistry. In addition, we found that downregulation of RACK1 expression using an RNA silencing approach in SKVO3 tumor cells significantly suppressed the proliferation, migration and invasion in vitro and tumor growth in vivo. Furthermore, it is found that RACK1 silencing was able to significantly suppress constitutive phosphorylation of Akt and MAPK, which may contribute to the inhibition of tumor growth. These results suggest that RACK1 can act as a new promising diagnostic biomarker and a potential anticancer therapeutic target for EOC.
\end{abstract}

\section{Introduction}

Epithelial ovarian cancer (EOC) is one of the most common fatal tumors of the female reproductive tract, and accounts for $90 \%$ of the incidence and the majority of deaths due to this malignancy (1-3). High mortality rates for ovarian cancer are mainly attributed to the late stage diagnosis of disease because almost 60-65\% of ovarian cancer cases are first diagnosed when cancer has already metastasized beyond the confines of the ovarian tissue. Despite the promise for better outcomes in recurrent EOC and advanced-stage ovarian cancer through chemosensitivity or biologic therapies, abdominal recurrence remains a serious clinical issue due to poor prognosis (4).

Correspondence to: Professor Tianmin Xu, Department of Gynaecology and Obstetrics, The Second Hospital of Jilin University, Changchun, Jilin 130041, P.R. China

E-mail: xutianmin518@sina.com

Key words: epithelial ovarian cancer, receptor of activated kinase 1, RNA silencing, tumorigenicity
Therefore, it is important to identify and develop specific markers to formulate novel therapeutic methods for advanced and recurrent ovarian cancer.

The receptor of activated C-kinase 1 (RACK1) was first identified as an anchoring protein for the conventional protein kinase C (PKC) (5). Several signal pathways were regulated by RACK1 interaction with protein kinases $\mathrm{C}$ family members, such as PKC $\alpha$ and PKC $\beta$ II (6-9). RACK1 has been involved in a series of cellular processes including regulation of protein translation (10,11), tissue development (12-14) cellular stress (15) and mammalian circadian clock (7). Importantly, it was expressed in some cancer cells and is involved in cancer progression (16-19). Shi et al found that RACK1 expression was upregulated in nonsmall cell lung cancer (NSCLC) samples, silencing RACK1 dramatically inhibited in vivo tumor growth and metastasis by blocking the sonic hedgehog signaling pathway (16). It has been reported that RACK1 downregulates levels of the proapoptotic protein Femlb in metastatic, apoptosis-resistant colon cancer cells, which may promote apoptosis-resistance during progression of colon cancer (17). Cao et al found that RACK1 is an independent prognosis-related factor and promotes breast carcinoma proliferation and invasion/metastasis in vitro and in vivo (18). These studies implied that RACK1 could promote carcinoma proliferation and invasion/metastasis, and the downregulated RACK1 was able to induce cancer cell apoptosis and inhibited cancer cell proliferation and migration. However, to our knowledge, the effect of RACK1 expression on tumorigenicity in ovarian cancer in vivo and in vitro has rarely been reported (19).

In this study, we investigate the effect of downregulating the RACK1 expression on cell growth, cell apoptosis, cell migration and tumor growth in human ovarian cancer in vivo and in vitro.

\section{Materials and methods}

Patients and immunohistochemistry. Paraffin-embedded tissue samples from 50 EOC patients, diagnosed at the Department of Obstetrics and Gynecology, The Second Affiliated Hospital of Jilin University (Changchun, China) from December 2010 to September 2012 were studied. Clinicopathological information was collected from clinical data of EOC patients (Table I). Tumors were staged according to the tumor node metastasis staging system of the International Union against Cancer (UICC). This study was approved by the ethics committee of 
Jilin Hospital and an informed consent was obtained from all participants.

To detect the expression and localization of RACK1 protein in ovarian tumor tissue, immunohistochemistry was performed using an SP reagent kit (Tiangen, Beijing, China) according to the manufacturer's instructions. The degree of immunoreactivity was assessed according to Campo et al (20). Immunoreactivity was measured semiquantitatively using a scale from 0 to 3 score, where score 0 represents no immunostaining, 1 represents fewer than $25 \%$ cells reactive, 2 represents $25-50 \%$ of the cells reactive and 3 represents more than $50 \%$ of the cells reactive. Values of 0 and 1 were considered to indicate negative staining, and 2 and 3 to indicate positive staining. Five cases with discordant results were re-evaluated to obtain agreement.

Cell culture. SKOV3 cells were obtained from the American Type Culture Collection (ATCC, Manassas, VA, USA) and cultured in modified RMPI-1640 medium (Sigma, St. Louis, MO, USA) supplemented with $10 \%$ fetal bovine serum (FBS, Gibco, Carlsbad, CA, USA), cells were maintained in a humidified $37^{\circ} \mathrm{C}$ incubator with $5 \% \mathrm{CO}_{2}$.

siRNA design and conduct. Templates of small interfering RNA (siRNA) against RACK1 were designed according to the instructions in the Silencer siRNA Construction kit (Ambion Inc., Austin, TX, USA) and synthesized (Invitrogen, Carlsbad, CA, USA). Sequences of the siRNA1 templates are: sense, 5'-AAGCAAGAAGTTATCAGTACCCCTGTCTC-3'; and antisense, 5'-AAGGTACTGATAACTTCTTGCCCTGTCTC-3'. Sequences for the scrambled control are: sense, 5'-CACAAGTC GAAACGTAAATGTCCTGTCTC-3'; and antisense, 5'-ACA TTTACGTTTCGACTTGTGCCTGTCTC-3'. Sequences of the siRNA2 templates are: sense, 5'-AACTATGGAATTCCAC AGCGTCCTGTCTC-3'; and antisense, 5'-AAACGCTGTGGA ATTCCATAGCCTGTCTC-3'. The cDNAs corresponding to the three siRNAs and NC were subcloned into the replicationdeficient, self-inactivating lentiviral expression vector pGCSILRNAi-GFP (Shanghai Gene Chem, Shanghai, China). Lentivirus containing siRNA was produced according to Coleman et al as previously described (21). The final titer of siRNA1 siRNA2 and negative control (NC) were $4 \times 10^{8}, 5 \times 10^{8}$ and $1 \times 10^{9} \mathrm{TU} / \mathrm{ml}$, respectively.

Cell viability analysis. MTT assay was used to determine the effect of siRNA-RACK1 on the proliferation of cells. SKOV3 cells were incubated at a concentration of $5 \times 10^{3}$ cell/well in a 96-well plate, and grown at $37^{\circ} \mathrm{C}, 5 \% \mathrm{CO}_{2}$ incubator until cell adherence. The cells on the culture plate were divided into groups on the basis of parallel lines after an overnight incubation in fresh RPMI-1640 containing $0.5 \%$ FBS, each group had 4 wells in one line for each group. After the 24-h attachment period, cells were treated with $5 \mu 1$ different siRNA and NC. A total of $20 \mu \mathrm{l}$ MTT $(5 \mathrm{mg} / \mathrm{ml})$ was added and the cells were incubated for another $4 \mathrm{~h}$ at the end of the treatment, $200 \mu \mathrm{l}$ of DMSO was added to each well after removing the supernatant. After shaking the plate for $10 \mathrm{~min}$, cell viability was obtained by measuring the absorbance at $490 \mathrm{~nm}$ wavelength by Enzyme-labeling Instrument (Bio-Tek ELX800, Winooski, VT, USA), this assay was done in triplicate. The inhibition rate
Table I. The correlation of RACK1 overexpression with clinicopathologic features of epithelial ovarian cancer.

\begin{tabular}{lccc}
\hline Clinical factors & Positive & Negative & P-value \\
\hline Mean age & & & \\
$<50(\mathrm{n}=20)$ & 14 & 6 & 0.944 \\
$\geq 50(\mathrm{n}=30)$ & 22 & 8 & \\
Metastasis & & & \\
No $(\mathrm{n}=26)$ & 15 & 3 & $<0.01$ \\
Yes $(\mathrm{n}=24)$ & 21 & & \\
Clinical stage & & 13 & $<0.01$ \\
I-II (n=35) & 22 & & \\
III-IV (n=15) & 14 & 8 & 0.457 \\
Tumor size & & 6 & \\
$<1.0 \mathrm{~cm}(\mathrm{n}=28)$ & 20 & & \\
$\geq 1.0 \mathrm{~cm}(\mathrm{n}=22)$ & 16 & 4 & 0.896 \\
Histological grade & & 6 & \\
G1 $(\mathrm{n}=15)$ & 11 & 4 & \\
G2 $(\mathrm{n}=24)$ & 18 & 7 &
\end{tabular}

was calculated according to the following formula (21): inhibition rate $(\%)=[1$ - (average absorbance of experimental group/ average absorbance of blank control group)] x $100 \%$.

Apoptosis analysis. SKOV3 cells were cultured in 6-well plates in RPMI-1640 with $10 \%$ FBS medium and were treated with different siRNA for 24,48 and $72 \mathrm{~h}$. The cover slips were washed three times with phosphate-buffered saline (PBS) and single cell suspensions were fixed in $1 \%$ PBS. Cells were stained with $100 \mu \mathrm{g} / \mathrm{ml}$ acridine orange (AO) and $100 \mu \mathrm{g} / \mathrm{ml}$ ethidium bromide (EB) for $1 \mathrm{~min}$. Then cells were observed under fluorescence microscope. At least 200 cells were counted and the percentage of apoptotic cells was determined. Triplicates were performed in all experiments, and experiments were performed five times.

Cell migration assay. The migration assay was performed using a 12-well Boyden Chamber (Neuro Probe, Gaithersburg, MD, USA) with an $8 \mu \mathrm{m}$ pore size. About $1 \times 10^{5}$ cells were seeded into the upper wells of the Boyden Chamber and incubated for $6 \mathrm{~h}$ at $37^{\circ} \mathrm{C}$ in medium containing $1 \% \mathrm{FBS}$. Medium containing $10 \%$ FBS was used as a chemoattractant in the lower wells. Cells that did not migrate through the pores of the Boyden Chamber were manually removed with a rubber instrument. Cells that migrated to the lower side of the membrane were stained with hematoxylin and eosin and photographed using an inverted microscope.

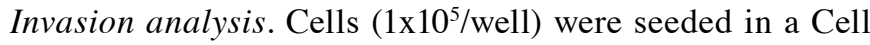
Culture Insert ( $8 \mu \mathrm{m}$ pore size; Falcon, BD Biosciences, San Jose, CA, USA), precoated with $25 \mu 1$ of $20 \%$ Matrigel (2-3 $\mathrm{mg} / \mathrm{ml}$ protein), and then placed in a 24 -well plate (Falcon, BD Biosciences). After cells had been cultured at $37^{\circ} \mathrm{C}$ for $40 \mathrm{~h}$, they were fixed and stained with $0.5 \%$ crystal violet. The cells on the top of the cell culture insert were removed 
by wiping with a cotton swab, and cell invasion was observed with an immunofluorescence microscope by counting the cells that had invaded into the lower of the cell culture insert. All experiments were performed in triplicate.

Tumor xenograft assay. All animal experiments were performed in accordance with institutional guidelines, following a protocol approved by the Ethics Committees of the Disease Model Research Center, The Second Hospital of Jilin University. About 6 weeks old female BALB mice were maintained under specific pathogen-free conditions and provided with food and water ad libitum. All the animals were fed with a normal pellet diet one week prior to the experimentation. In vitro cultured SKOV3 cells were injected s.c. into the right supra scapula region of the mice. Tumor volume was calculated by formula: volume $=$ length $\mathrm{x}$ width ${ }^{2} / 2$. When tumors grew to an average volume of $75 \mathrm{~mm}^{3}$, mice were randomly divided into siRNA1, siRNA2, NC group ( $\mathrm{n}=10$ in each) and by administered siRNA1, siRNA2, and NC plus PBS in a total volume of $15 \mu 1$ (5 $\mu 1$ virus plus $10 \mu \mathrm{l}$ PBS) one time a week for 21 days, respectively. When control mice started to succumb to their tumors, the mice in all treatment groups were euthanized. After the mice had been sacrificed, the tumors were removed and directly embedded in an optimal cutting temperature (OCT) compound at $-80^{\circ} \mathrm{C}$.

Real-time quantitative PCR. Total RNA was isolated from SKOV3 cell lines and ovarian tissues of EOC patients using TRIzol reagent (Invitrogen) according to the manufacturer's instructions. RNA was reverse-transcribed into cDNA using a Primescript $^{\mathrm{TM}} \mathrm{RT}$ reagent kit according to the manufacturer's instructions. Real-time quantitative polymerase chain reaction (PCR) was performed with the SYBR-Green fluorescent dye method, and a Rotor Gene 3000 real-time PCR apparatus. Human primer sequences were: RACK1 forward, 5'-TCTCTTT CCAGCGTGGCCATTAGA-3' and reverse, 5'-CCTCGAAGC TGTAGAGATTCCGACAT-3'; $\beta$-actin forward, 5'-GATCAT TGCTCCTCCTGAGC-3' and reverse, 5'-ACTCCTGCTTGC TGATCCAC-3'. The PCR conditions were as follows: pre-denaturing at $95^{\circ} \mathrm{C}$ for $2 \mathrm{~min}$, followed by 45 cycles of denaturation at $95^{\circ} \mathrm{C}$ for $10 \mathrm{sec}$, annealing/extension at $60^{\circ} \mathrm{C}$ for $20 \mathrm{sec}$. The amplification specificity was checked by melting curve analysis. The $2^{-\triangle \triangle C T}$ method was used to calculate the relative abundance of target gene expression generated by Rotor-Gene Real-Time Analysis Software 6.1.81. For each cDNA, the target gene mRNA level was normalized to $\beta$-actin mRNA level. The experiments were performed three times.

Western blot analysis. Cultured cells were washed twice with PBS and lysed in radioimmune precipitation assay buffer for $30 \mathrm{~min}$ on ice. Cell lysates were clarified by centrifugation $(10,000 \mathrm{x} \mathrm{g}, 15 \mathrm{~min})$, and protein concentrations were determined using the Bradford reagent (Sigma). Lysates were separated on 8 or 15\% SDS-PAGE; proteins were transferred to Immobilon membrane (Millipore, Bedford, MA, USA) immunoblotted with specific primary antibodies and incubated with corresponding horseradish peroxidase-conjugated secondary antibody. The primary antibodies used in the western blots were: antibodies against Akt, phosphorylated (p)-Akt, MAPK, p-MAPK, anti-RACK1 antibody (Santa Cruz Biotechnology,


Figure 1. Levels of RACK1 are increased in EOC clinical samples. (A) Quantitative real-time PCR results of relative expression level of RACK1 in 50 cases of EOC and normal ovarian samples are shown. The mRNA expression level of $\beta$-actin was quantified as an internal standard and used to normalize the level of RACK1 from the same sample. (B) Immunohistochemical results of RACK1 expression in EOC and matched normal ovarian tissues. In cancer tissue from individuals with EOC, intense RACK1 immunoreactivity (yellow) was observed. In corresponding paired normal tissues, however, RACK1 expression was weaker. ${ }^{*} \mathrm{P}<0.05,{ }^{* * *} \mathrm{P}<0.01$ vs. control.

Santa Cruz, CA, USA). All immunoblots were visualized by enhanced chemiluminescence (Pierce, Rockford, IL, USA).

Statistical analysis. The data are expressed as mean \pm SEM. Statistical analysis between two samples was performed using Student's t-test. Statistical comparison of more than two groups was performed using one-way ANOVA followed by a Tukey's post hoc test and considered significant at ${ }^{*} \mathrm{P}<0.05$ or ${ }^{* *} \mathrm{P}<0.01$.

\section{Results}

RACK1 is upregulated in EOC and correlates with clinical features of EOC patients. To identify the potential roles of RACK1 in the development and progression of EOC, we assessed its expression level by real-time polymerase chain reaction (PCR) in 50 pairs of matched ovarian tissue samples. Expression levels of RACK1 were significantly higher in EOC tumors compared with their normal ovarian counterparts (Fig. 1A). Furthermore, elevated levels of RACK1 protein were found in EOC tumors compared with the paired normal tissues from the same patients as shown by immunochemical staining (Fig. 1B). Further analysis revealed that the upregulation of 
A



B

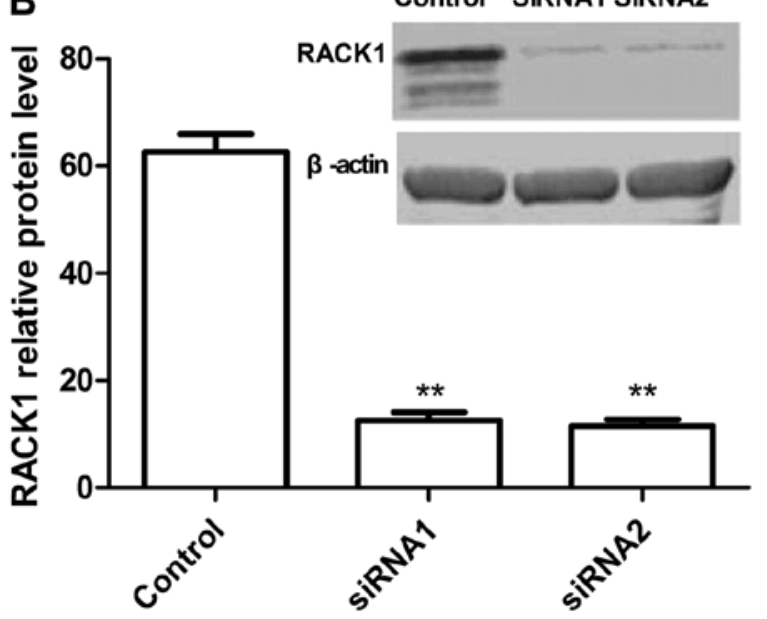

Figure 2. Silencing RACK1 by siRNA inhibits the RACK1 expression in SKVO3 tumor cells. (A) Quantitative real-time PCR analysis of RACK1 after RNAi silencing. (B) Western blot analysis of RACK1 after RNAi silencing. The images are representative results from three independent experiments. ${ }^{*}<0.05$, ${ }^{* *} \mathrm{P}<0.01$ vs. control.
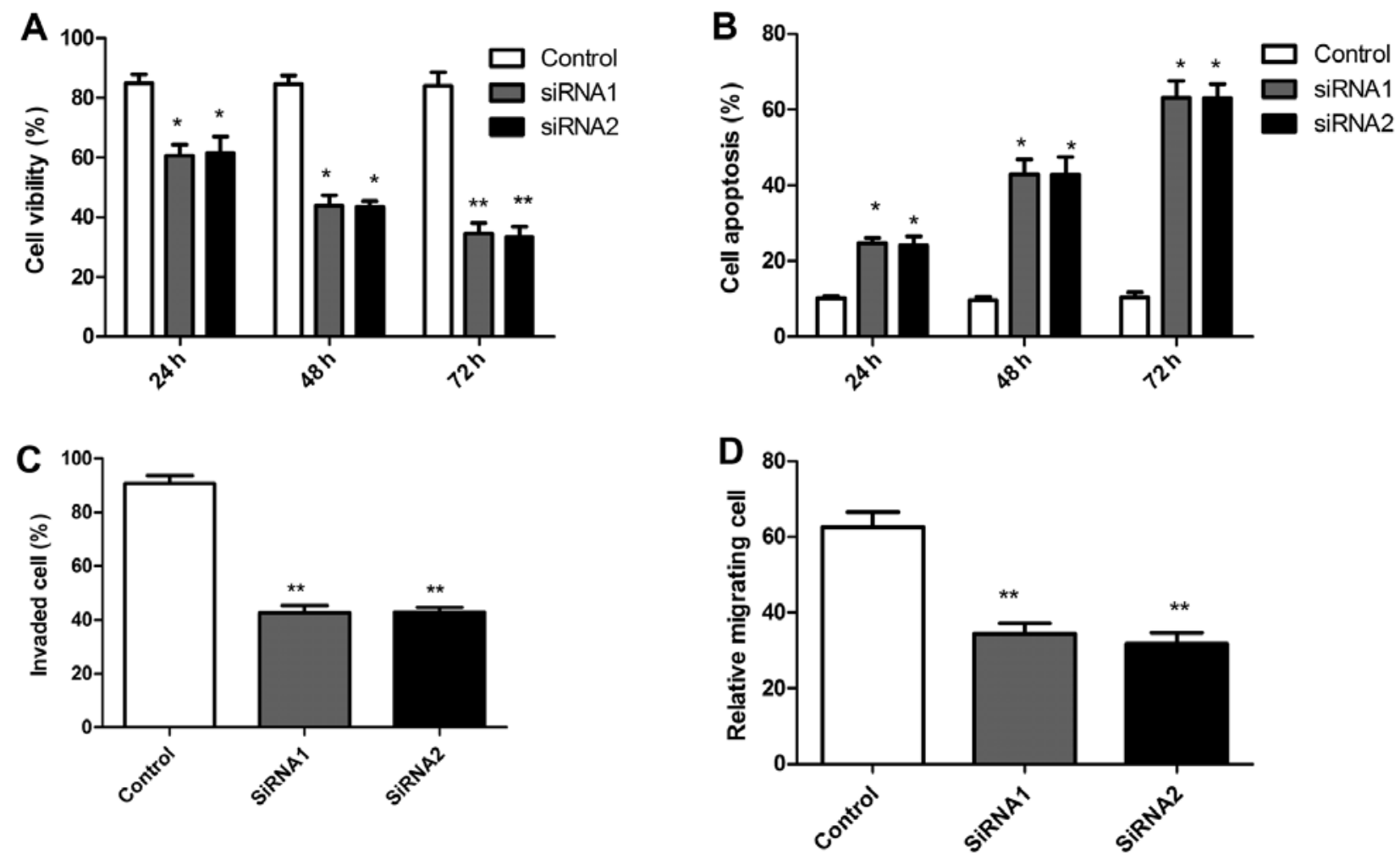

Figure 3. RACK1 silencing inhibits tumor cell growth and invasion and induces cell apoptosis in vitro. SKOV3 cells were transfected with scrambled control siRNA (control) or RACK1 siRNAs (SiRNA1 and SiRNA2), as indicated. Downregulation of RACK1 by transfection of siRNA significantly (A) suppressed the proliferation, (B) induced cell apoptosis, and significantly suppressed (C) migration and (D) invasive ability of SKVO3 cells, ${ }^{*} \mathrm{P}<0.05,{ }^{* *} \mathrm{P}<0.01 \mathrm{vs}$. control.

RACK1 protein in EOC samples was positively correlated with both lymph node metastasis and tumor TNM stage $(\mathrm{P}<0.01$, Table I). No correlation occurred between RACK1 protein levels, age, tumor size and histologic grade. These data suggested that RACK1 expression correlates with advanced EOC.

siRACK1 transformed into lentiviral vector efficiency assay. To understand the function of RACK1 in EOC, we used the RNAi- mediated knockdown lentiviral vector to decrease the basal level of RACK1 (knockdown of RACK1) in SKOV3 cells by real-time RT-PCR and western blot analysis. Real-time RT-PCR and western blot analysis results showed that two independent target sequences siRNA1 and siRNA2 markedly decreased the expression of RACK1 compared with the control sequence (Fig. 2). These results demonstrated that siRNA targeting RACK1 significantly silenced RACK1 expression in vitro. 

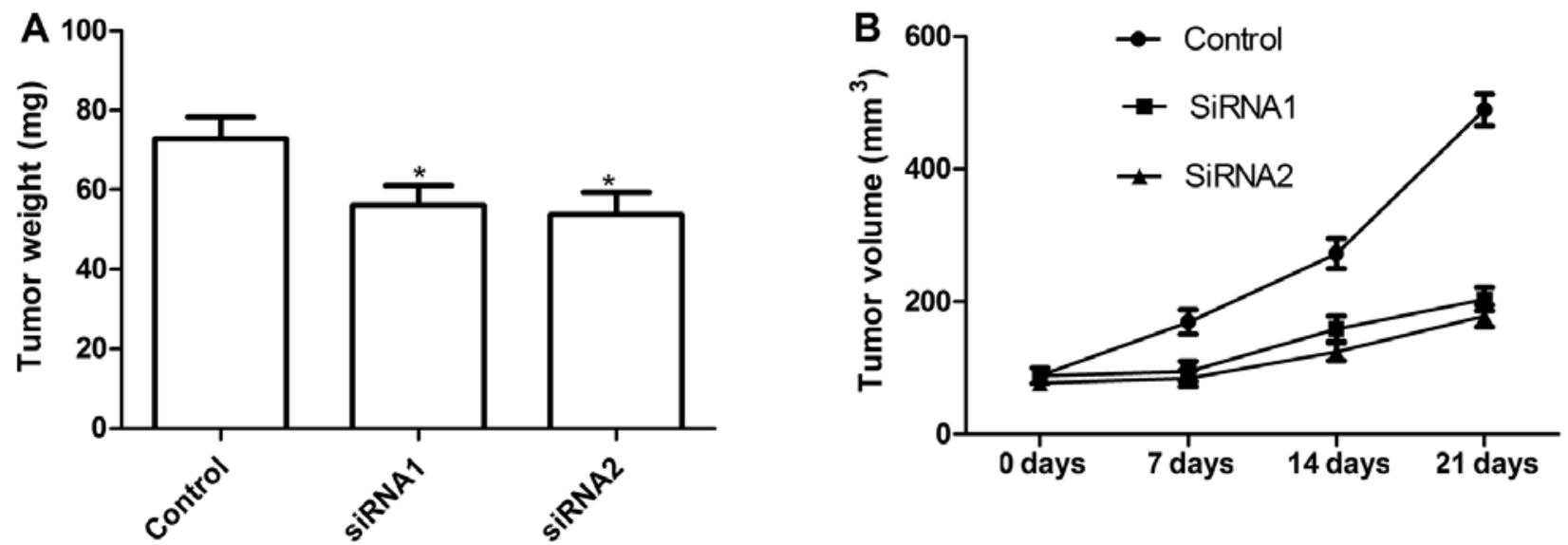

Figure 4.RACK1 silencing significantly suppressed tumor growth in vivo.(A) Tumor weight in xenograft mice 21 days after silencing the RACK1. (B) Representative images of xenograft tumor models and tumor growth curves of SKVO3 cells, ${ }^{*} \mathrm{P}<0.05,{ }^{* * *} \mathrm{P}<0.01$ vs. control.

Silencing RACK1 in ovarian cancer cells reduces proliferation, migration and invasion in vitro. Using these two siRNAs, we examined the effects of RACK1 silencing on tumor cell growth in vitro. The anti-proliferative effect of RACK1 silencing on SKOV3 tumor cell were examined using MTT assays. It was found that it significantly inhibited the proliferation of SKOV3 tumor cells compared with control cells (scrambled siRNA) (Fig. 3A). Conversely, RACK1 silencing significantly improved apoptosis of SKOV3 tumor cells compared with control time-dependently, as determined using the AO staining assay (Fig. 3B). Furthermore, the effects of silencing RACK1 on ovarian tumor cell migration and invasion were assessed. As shown in Fig. 3C, silencing RACK1 reduced migration capacity in SKOV3 tumor cells. In addition, cell invasion ability was significantly decreased, when assessed after $48 \mathrm{~h}$ by the modified Boyden chamber assays (Fig. 3D). Together, these data indicate that RACK1 plays an important regulatory role in tumor cell growth and migration.

Silencing RACK1 suppresses tumor growth in vivo. To obtain definitive evidence that RACK1 is required for ovarian tumor growth in vivo, we conducted a xenograft assay by injecting the control (si-scrambled) and RACK1-silenced tumor cells subcutaneously into mice and compared the growth rate to solid tumors. Mice were sacrificed and taken out tumor tissue 21 days after treatment. Then tumor weight of the animals was measured. The tumor weight of siRNA group was lower than those of control group (Fig. 4A). We found that tumor volume after RACK1 silencing was significantly slower in SKOV3 tumor cells compared with control cells (Fig. 4B). These results indicate that suppression of RACK1 expression in ovarian tumor cells markedly suppresses their tumorigenicity in mice.

RACK1 silencing inhibites the phosphorylation of Akt and MAPK in vitro. To clarify the molecular mechanisms involved due to downregulation of RACK1 inhibition the growth of human tumor xenografts, in the present study, we focused on the effects of RACK1 silencing on the activation of MAPK, Akt, which participate in the main intracellular signaling

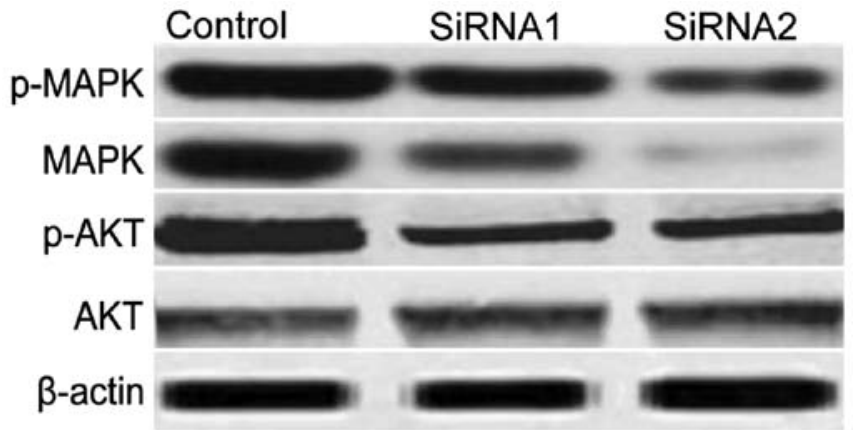

Figure 5. Effect of RACK1 silencing on the phosphorylation of Akt and MAPK in vitro. SKVO3 cells were transfected with scrambled control siRNA or RACK1 siRNAs and the cell lysates were subjected to western blot analysis. Downregulation of RACK1 by siRNA resulted in significant suppression of Akt and MAPK; p, phosphorylated.

required for cell proliferation and survival. As shown in Fig. 5, compared with control siRNAs, RACK1 silencing led to a significant suppression of Akt and MAPK activation. These results indicate that RACK1 silencing inhibits tumor cell growth, to some extent, by suppressing MAPK and Akt-mediated signaling.

\section{Discussion}

RACK1, as a crucial scaffold protein, was originally cloned as an anchoring protein for PKC; it plays a critical role in stabilizing the active form of $\mathrm{PKC}$ and in permitting its translocation to different sites within a cell $(22,23)$. A large number of literature reveals that RACK1 can associate with various signaling molecules and is involved in the regulation of various cell functions (24-27). Increased RACK1 expression has been reported in lung, colon, breast and hepatocellular carcinomas and human melanomas (28-30). Zhang et al found that increased RACK1 plays an important role in IR/IGF-1R-mediated promotion of anchorage-independent growth of certain ovarian cancer cells (19). However, this study did not focus on RACK1 expression in EOC patients. To our knowledge, in the present study, we 
first found that RACK1 was elevated in most EOC compared to normal ovarian tissue, and its expression level correlated with key pathological characteristics including clinical stage and metastasis. Our findings also show that downregulation of RACK1 results in the inhibition of proliferation migration for SKVO3 cells in vitro, and suppression of solid tumor growth in vivo. These results provide evidence that RACK1 is required for tumor growth and that it may be a diagnostic marker.

Previous studies showed that several signal pathways were regulated by RACK1 interaction with protein kinases C family members, such as PKC, Src and IGF-1R $(19,22,26)$. It is widely accepted, that aberrant activation of several PKC isozymes leads to tumorigenesis; thus, as an important stabilizer of PKC activity, RACK1 may play a pivotal role in regulating PKC signaling-related tumor progression $(22,23,31)$. Berns et al showed that RACK1 is upregulated in vascular endothelial cells during angiogenesis and may contribute to tumor growth and spreading (30). Wang et al found that RACK1 silencing significantly attenuated tumorassociated angiogenesis by inhibiting the expression of two critical angiogenic factors (17). Furthermore, Keily et al showed that the proliferation of MCF-7 cells was enhanced by overexpression of RACK1, whereas IGF-1-mediated protection from etoposide-dependent death was greatly reduced (32). Two other groups also reported that knockdown of RACK1 suppressed IGF-1R and androgen receptordependent tumor cell growth $(33,34)$. Zhang et al further showed that RACK1 acts as an adaptor protein to facilitate the association of STAT3 with IR/IGF-1R and subsequent phosphorylation and activation of STAT3 specifically (19). Shi et al found that RACK1 activated the sonic hedgehog signaling pathway by interacting with and activating smoothened to mediate Gli1-dependent transcription in NSCLC cells (16). These studies show that RACK1 is simultaneously involved in widespread signaling pathways owing to its association with PKC, Src family members, and other multiple pathways related to these proteins appear to be activated in the process of tumor growth. Thus, RACK1 is implicated in a complicated signaling network rather than a simple pathway. In the present study, we focused on the effect of RACK1 on the activation of central players in growth signaling, such as Akt, MAPK, which are often constitutively activated in a variety of cancers and participated in the main intracellular signaling required for cell proliferation and survival $(35,36)$. We found that RACK1 silencing significantly inhibited the phosphorylation of Akt and MAPK to some extent in SKVO3 cells. Which implied that the suppression of Akt- or MAPKrelated pathways contributes, at least in part, to the inhibition of tumor growth.

A series of experiments (in vitro and in vivo) were designed to investigate the characteristics of RACK1 and to evaluate its role in ovarian tumorigenesis. Our results show that the silencing of RACK1 induced SKOV3 tumor cell apoptosis, which agrees with the downregulation of RACK1 inducing cell apoptosis in NSCLC (16). However, in contrast with a previous report that overexpression of RACK1 induced apoptosis of HT-29 colon carcinoma cells, partly by inhibiting Src activity (37). Moreover, recent studies showed that RACK1 knockdown inhibited cell growth but did not affect cell apoptosis $(17,18)$. These above studies suggested that RACK1 has opposing effects on cell apoptosis of different types of tumor cells, and the discrepancy may arise from differences the cell types used or in the experimental conditions.

In conclusion, to our knowledge, this is the first full-scale report concerning the association of RACK1 with ovarian carcinoma. The present study demonstrates that RACK1 was elevated in most EOC and its expression level correlated with key pathological characteristics including clinical stage and metastasis and it plays a critical role in tumor growth by affecting cell proliferation and tumor growth in vitro and in vivo. Based on the multiple functions of RACK1 in tumor growth, it may be considered a marker for diagnosis and a potential anticancer therapeutic target.

\section{References}

1. Jemal A, Bray F, Center MM, Ferlay J, Ward E and Forman D: Global cancer statistics. CA Cancer J Clin 61: 69-90, 2011.

2. Bast RC Jr, Hennessy B and Mills GB: The biology of ovarian cancer: new opportunities for translation. Nat Rev Cancer 9: 415-428, 1996.

3. Bowtell DD: The genesis and evolution of high-grade serous ovarian cancer. Nat Rev Cancer 10: 803-808, 2010.

4. Vaughan S, Coward JI, Bast RC Jr, Berchuck A, Berek JS, Brenton JD, Coukos G, Crum CC, Drapkin R, Etemadmoghadam D, Friedlander M, Gabra H, Kaye SB, Lord CJ, Lengyel E, Levine DA, McNeish IA, Menon U, Mills GB, Nephew KP, Oza AM, Sood AK, Stronach EA, Walczak H, Bowtell DD and Balkwill FR: Rethinking ovarian cancer: recommendations for improving outcomes. Nat Rev Cancer 11: 719-725, 2011.

5. Csukai M and Mochly-Rosen D: Pharmacologic modulation of protein kinase C isozymes: the role of RACKs and subcellular localisation. Pharmacol Res 39: 253-259, 1999.

6. Calura E, Cagnin S, Raffaello A, Laveder P, Lanfranchi G and Romualdi C: Meta-analysis of expression signatures of muscle atrophy. Gene interaction networks in early and late stages. BMC Genomics 9: 630, 2008.

7. Robles MS, Boyault C, Knutti D, Padmanabhan K and Weitz CJ: Identification of RACK1 and protein kinase $\mathrm{C}$ as integral components of the mammalian circadian clock. Science 327: 463-466, 2010

8. Li CL, Toda K, Saibara T, Zhang T, Ono M, Iwasaki S, Maeda T, Okada T, Hayashi Y, Enzan H, Shizuta Y and Onishi S: Estrogen deficiency results in enhanced expression of smoothened of the Hedgehog signaling in the thymus and affects thymocyte development. Int Immunopharmacol 2: 823-833, 2003.

9. Bible KC, Suman VJ, Molina JR, Smallridge RC, Maples WJ, Menefee ME, Rubin J, Sideras K, Morris JC III, McIver B, Burton JK, Webster KP, Bieber C, Traynor AM, Flynn PJ, Goh BC, Tang H, Ivy SP and Erlichman C: Efficacy of pazopanib in progressive, radioiodine-refractory, metastatic differentiated thyroid cancers. Results of a phase 2 consortium study. Lancet Oncol 11: 962-972, 2010.

10. Toftgard R: Hedgehog signaling in cancer. Cell Mol Life Sci 57: $1720-1731,2000$

11. Gialmanidis IP, Bravou V, Amanetopoulou SG, Varakis J, Kourea $\mathrm{H}$ and Papadaki $\mathrm{H}$ : Overexpression of hedgehog pathway molecules and FOXM1 in non-small-cell lung carcinomas. Lung Cancer 66: 64-74, 2009.

12. Kadrmas JL, Smith MA and Pronovost SM: Characterization of RACK1 function in Drosophila development. Dev Dyn 236: 2207-2215, 2007.

13. Mor I, Sklan EH, Podoly E, Pick M, Kirschner M, Yogev L, Bar-Sheshet Itach S, Schreiber L, Geyer B, Mor T, Grisaru D and Soreq H: Acetylcholinesterase-R increases germ cell apoptosis but enhances sperm motility. J Cell Mol Med 12: 479-495, 2008.

14. Battaini F, Pascale A, Paoletti R and Govoni S: The role of anchoring protein RACK1 in PKC activation in the ageing rat brain. Trends Neurosci 20: 410-415, 1997.

15. Qiu Y, Mao T, Zhang Y, Shao M, You J, Ding Q, Chen Y, Wu D, Xie D, Lin X, Gao X, Kaufman RJ, Li W and Liu Y: A crucial role for RACK1 in the regulation of glucose-stimulated IRE1 activation in pancreatic cells. Sci Signal 3: ra7, 2010. 
16. Shi S, Deng YZ, Zhao JS, Ji XD, Shi J, Feng YX, Li G, Li JJ, Zhu D, Koeffler HP, Zhao Y and Xie D: Sonic Hedgehog signaling pathway cancer tumorigenicity through activating RACK1 promotes non-small-cell lung. J Biol Chem 287: 7845-7858, 2012.

17. Wang F, Osawa $\mathrm{T}$, Tsuchida R, Yuasa $\mathrm{Y}$ and Shibuya $\mathrm{M}$ : Downregulation of receptor for activated C-kinase 1 (RACK1) suppresses tumor growth by inhibiting tumor cell proliferation and tumor-associated angiogenesis. Cancer Sci 102: 2007-2013, 2011.

18. Cao XX, Xu JD, Xu JW, Liu XL, Cheng YY, Wang WJ, Li QQ, Chen Q, Xu ZD and Liu XP: RACK1 promotes breast carcinoma proliferation and invasion/metastasis in vitro and in vivo. Breast Cancer Res Treat 123: 375-386, 2010.

19. Zhang W, Zong CS, Hermanto U, Lopez-Bergami P, Ronai Z and Wang LH: RACK1 recruits STAT3 specifically to insulin and insulin-like growth factor 1 receptors for activation, which is important for regulating anchorage independent growth. Mol Cell Biol 26: 413-424, 2006.

20. Campo E, Merino MJ, Liotta L, Neumann R and Stetler-Stevenson W: Distribution of 72-kd type IV collagenase in nonneoplastic and neoplastic thyroid tissue. Hum Pathol 23: 1395-1401, 1992.

21. Coleman JE, Huentelman MJ, Kasparov S, Metcalfe BL, Paton JF, Katovich MJ, Semple-Rowland SL and Raizada MK: Efficient large-scale production and concentration of HIV-1-based lentiviral vectors for use in vivo. Physiol Genomics 12: 221-228, 2003.

22. Ron D, Chen CH, Caldwell J, Jamieson L, Orr E and Mochly-Rosen D: Cloning of an intracellular receptor for protein kinase $\mathrm{C}$ : a homolog of the beta subunit of $\mathrm{G}$ proteins. Proc Natl Acad Sci USA 91: 839-843, 1994.

23. Ron D and Mochly-Rosen D: Agonists and antagonists of protein kinase $\mathrm{C}$ function, derived from its binding proteins. J Biol Chem 269: 21395-21398, 1994.

24. Liliental J and Chang DD: Rack1: a receptor for activated protein kinase $\mathrm{C}$, interacts with integrin beta subunit. J Biol Chem 273: 2379-2383, 1998.

25. Wang F, Yamauchi M, Muramatsu M, Osawa T, Tsuchida R and Shibuya M: RACK1 regulates VEGF/Flt1-mediated cell migration via activation of a PI3K/Akt pathway. J Biol Chem 286: 9097-9106, 2011

26. Wang F, Yamauchi M, Muramatsu M, Osawa T, Tsuchida R and Shibuya M: The interaction of Src and RACK1 is enhanced by activation of protein kinase $\mathrm{C}$ and tyrosine phosphorylation of RACK1. J Biol Chem 276: 20346-20356, 2001.
27. Besson A, Wilson TL and Yong VW: The anchoring protein RACK1 links protein kinase $\mathrm{C}$ epsilon to integrin beta chains. Requirements for adhesion and motility. J Biol Chem 277: 22073-22084, 2002.

28. Bourd-Boittin K, LePabic H and Bonnier D: RACK1, a new ADAM12 interacting protein. Contribution to liver fibrogenesis. J Biol Chem 283: 26000-26009, 2008.

29. Egidy G, Jule S and Bosse P: Transcription analysis in the MeLiM swine model identifies RACK1 as a potential marker of malignancy for human melanocytic proliferation. Mol Cancer 7: 34, 2008.

30. Berns H, Humar R, Hengerer B, Kiefer FN and Battegay EJ: RACK1 is upregulated in angiogenesis and human carcinomas. FASEB J 14: 2549-2558, 2000.

31. Schechtman D and Mochly-Rosen D: Adaptor proteins in protein kinase $\mathrm{C}$ mediated signal transduction. Oncogene 20: 6339-6347, 2001

32. Kiely PA, Sant A and O'Connor R: RACK1 is an insulin-like growth factor 1 (IGF-1) receptor-interacting protein that can regulate IGF-1-mediated Akt activation and protection from cell death. J Biol Chem 277: 22581-22589, 2002.

33. Kraus S, Gioeli D, Vomastek T, Gordon V and Weber MJ: Receptor for activated C kinase 1 (RACK1) and Src regulate the tyrosine phosphorylation and function of the androgen receptor. Cancer Res 66: 11047-11054, 2006.

34. Hermanto U, Zong CS, Li W and Wang LH: RACK1, an insulin-like growth factor I (IGF-I) receptor-interacting protein, modulates IGF-I-dependent integrin signaling and promotes cell spreading and contact with extracellular matrix. Mol Cell Biol 22: 2345-2365, 2002.

35. Falasca M: PI3K/Akt signalling pathway specific inhibitors: a novel strategy to sensitize cancer cells to anti-cancer drugs. Curr Pharm Des 16: 1410-1416, 2010.

36. Katz M, Amit I and Yarden Y: Regulation of MAPKs by growth factors and receptor tyrosine kinases. Biochim Biophys Acta 1773: 1161-1176, 2007.

37. Mamidipudi V, Dhillon NK, Parman T, Miller LD, Lee KC and Cartwright CA: RACK1 inhibits colonic cell growth by regulating Src activity at cell cycle checkpoints. Oncogene 26: 2914-2924, 2007. 